Published in final edited form as:

Eur J Nucl Med Mol Imaging. 2016 March ; 43(3): 474-481. doi:10.1007/s00259-015-3179-2.

\title{
Vesicular Monoamine Transporter Protein Expression Correlates with Clinical Features, Tumor Biology, and MIBG Avidity in Neuroblastoma: A Report from the Children's Oncology Group
}

\author{
William Temple ${ }^{\# 1,4}$, Lori Mendelsohn ${ }^{\# 1,4}$, Grace E. Kim² ${ }^{2}$, Erin Nekritz ${ }^{1,4}$, W. Clay \\ Gustafson ${ }^{1,4}$, Lawrence Lin $^{3}$, Kathy Giacomini ${ }^{3}$, Arlene Naranjo ${ }^{5}$, Collin Van Ryn ${ }^{5}$, Gregory \\ A. Yanik ${ }^{6}$, Susan G. Kreissman ${ }^{7}$, Michael Hogarty ${ }^{8}$, Katherine K. Matthay ${ }^{1,4}$, and Steven G. \\ DuBois ${ }^{1,4}$ \\ ${ }^{1}$ Department of Pediatrics, UCSF School of Medicine, San Francisco, CA 94143 \\ ${ }^{2}$ Department of Pathology, UCSF School of Medicine, San Francisco, CA 94143 \\ ${ }^{3}$ Department of Bioengineering and Therapeutic Sciences, UCSF School of Pharmacy, San \\ Francisco, CA 94143 \\ ${ }^{4}$ UCSF Benioff Children's Hospital, San Francisco, CA 94158 \\ ${ }^{5}$ Children's Oncology Group Statistics and Data Center, University of Florida, Gainesville, FL \\ 32607 \\ ${ }^{6}$ CS Mott Children's Hospital, University of Michigan, Ann Arbor, MI 48109 \\ ${ }^{7}$ Duke University Medical Center, Durham, NC 27710 \\ ${ }^{8}$ Children's Hospital of Philadelphia and Perelman School of Medicine, University of \\ Pennsylvania, Philadelphia, PA 19104. \\ \# These authors contributed equally to this work.
}

\begin{abstract}
Purpose-Vesicular monoamine transporters 1 and 2 (VMAT1 and VMAT2) are thought to mediate MIBG uptake in adult neuroendocrine tumors. In neuroblastoma, the norepinephrine transporter (NET) has been investigated as the principal MIBG uptake protein, though some tumors without NET expression concentrate MIBG. We investigated VMAT expression in neuroblastoma and correlated expression with MIBG uptake and clinical features.
\end{abstract}

Methods-We evaluated VMAT1 and VMAT2 expression by immunohistochemistry (IHC) in neuroblastoma tumors from 76 patients with high-risk metastatic disease treated on a uniform cooperative group trial (COG A3973). All patients had baseline MIBG diagnostic scans centrally

\footnotetext{
Corresponding Author: Steven DuBois, MD, UCSF School of Medicine, $55016^{\text {th }}$ Street, $4^{\text {th }}$ Floor, San Francisco, CA 94158 , Telephone: 415-476-3831, Facsimile: 415-476-5353, duboiss@peds.ucsf.edu. Conflict of Interest: The authors declare that they have no conflict of interest.

Ethical Approval: All procedures performed in studies involving human participants were in accordance with the ethical standards of the institutional and/or national research committee and with the 1964 Helsinki declaration and its later amendments or comparable ethical standards. For this type of study, formal consent is not required.
} 
reviewed. IHC results were scored as the product of intensity grading (0-3+) and percent of tumor cells expressing the protein of interest. Association of VMAT1 and VMAT2 scores with clinical and biological features was tested using Wilcoxon rank-sum tests.

Results-Patient characteristics were typical of high-risk neuroblastoma, though the cohort was intentionally enriched for patients with MIBG non-avid tumors $(n=20)$. VMAT1 and VMAT2 were expressed in $62 \%$ and $75 \%$ of neuroblastoma tumors, respectively. VMAT1 and VMAT2 scores were both significantly lower in MYCN amplified tumors and in tumors with high mitotic karyorrhectic index. MIBG avid tumors had significantly higher VMAT2 scores compared to MIBG non-avid tumors (median 216 vs. 45; $\mathrm{p}=0.04$ ). VMAT1 expression did not correlate with MIBG avidity.

Conclusions-VMAT1 and VMAT2 are expressed in the majority of neuroblastomas. Expression correlates with other biological features. Expression level of VMAT2 but not VMAT1 correlates with avidity for MIBG.

\section{Introduction}

Neuroblastoma is the most common pediatric extra-cranial solid tumor, with approximately 650 new cases each year in the United States [1]. More than half of children with neuroblastoma present with widespread metastatic disease at diagnosis, with a poor probability of long-term survival. Neuroblastoma most commonly presents in the adrenal medulla, but primary tumors can arise elsewhere along any portion of the sympathetic chain.

Neuroblastoma primary and metastatic tumors can be detected using an analogue of norepinephrine, metaiodobenzylguanidine (MIBG) tagged with a radionuclide [2]. Lowdose ${ }^{123}$ I-MIBG is used diagnostically to localize tumors, while high-dose ${ }^{131}$ I-MIBG is used as a targeted radiopharmaceutical [3]. In patients with relapsed or refractory neuroblastoma, high-dose ${ }^{131}$ I-MIBG has a response rate of 30-40\% [4]. Eventually ${ }^{131}$ IMIBG may play a role in the initial treatment of high-risk neuroblastoma and further understanding of mediators of MIBG uptake by neuroblastoma is critical.

Clinically, $90 \%$ of neuroblastomas are able to accumulate MIBG [5]. The remaining $10 \%$ of patients who present with neuroblastoma tumors that do not accumulate MIBG are currently not candidates for targeted imaging or therapy with MIBG. The norepinephrine transporter (NET) has been shown to facilitate specific uptake of MIBG, a norepinephrine analogue [6-8]. Clinically, our group and others have shown that there is a significant correlation between tumor NET expression and MIBG accumulation [9-11]. Our group has also identified tumors that are MIBG-avid but have little or no NET expression, and other tumors that express high levels of NET but do not accumulate MIBG [10]. These findings suggest that there are alternative mechanisms by which MIBG is specifically taken up and/or retained by neuroblastoma.

We hypothesized that the vesicular monoamine transporters (VMATs) might be expressed in neuroblastoma and play a role in mediating MIBG accumulation. VMATs are acidic glycoproteins with 12 transmembrane domains. There are two isoforms, VMAT1 and VMAT2 [12]. VMATs transport monoamine neurotransmitters, including norephineprine, 
against their concentration gradient from the cytosol into transport vesicles in the presynaptic neuron. Several pieces of evidence argue that MIBG is a substrate for VMATs. Reserpine, a VMAT inhibitor, hastens MIBG clearance from normal adrenal medulla and pheochromocytoma and reduces MIBG uptake in pheochromocytoma [13, 14, 7]. In neuroendocrine tumors other than neuroblastoma, VMAT expression has been shown to correlate with MIBG uptake [15, 14].

We conducted a retrospective study to describe VMAT1 and VMAT2 expression in neuroblastoma cell lines and human tumors. We further aimed to correlate VMAT1 and VMAT2 expression with clinical and biological features in patients with high-risk neuroblastoma, including MIBG avidity. Finally, we sought to assess the association between VMAT1 and VMAT2 expression with NET protein expression in these tumors.

\section{Materials and Methods}

\section{Western Blot for NET, VMAT1, and VMAT2 expression}

Neuroblastoma and control cells were grown in Iscove's modified Dulbecco's medium with $20 \%$ fetal bovine serum (FBS) + ITS or in Dulbecco's modified eagle medium with $10 \%$ FBS. HEK293T cells transfected to overexpress VMAT1, VMAT2, NET, or empty vector served as controls. Cells were harvested and lysed with Cell Signaling lysis buffer $+1 \%$ sodium dodecyl sulfate, sonicated, and supernatants boiled in lithium dodecyl sulfate sample buffer (Invitrogen). Protein was quantitated by bicinchoninic acid (BCA) assay (Pierce) and analyzed on 4-12\% gradient Bis-Tris gels (Invitrogen). Membranes were incubated overnight at $4{ }^{\circ} \mathrm{C}$ with primary antibodies [VMAT1 (abcam), VMAT2 (OriGene), hNET (MAb Technologies), MYCN (abcam), MYC (Cell Signaling), and GAPDH (Millipore)], then developed using horseradish peroxidase (HRP)-conjugated secondary antibodies (Cell Signaling) and ECL reagents (Amersham).

\section{Patients and MIBG Diagnostic Scans}

All patients had high-risk metastatic neuroblastoma and participated in Children's Oncology Group (COG) trial A3973 [16]. All patients were unique from our prior cohort [10], except one MIBG non-avid patient is included in both cohorts. Pathology, ploidy, 1p and 11q status, and $M Y C N$ copy number were determined in the COG central reference laboratory. Patients underwent diagnostic ${ }^{123} \mathrm{I}$ - or ${ }^{131} \mathrm{I}$-MIBG whole body scans at the time of study entry. Scans were obtained according to local institutional practice. Baseline diagnostic MIBG scans were available for central review for all patients included in this analysis [17]. The primary clinical endpoint for the current study, MIBG avidity, is based upon the results of this independent central review that preceded determination of tumor transporter expression levels for the current study. Among patients with MIBG-avid tumors, intensity of MIBG avidity was not scored.

This retrospective study was approved by the Committee on Human Research at the University of California, San Francisco. 


\section{Immunohistochemistry for NET, VMAT1, and VMAT2 Protein Expression}

Unstained slides of paraffin-embedded tumor material were obtained from the COG Neuroblastoma Reference Laboratory (Nationwide Children's Hospital, Columbus, $\mathrm{OH}$ ).

Slides were selected by the staff at the reference laboratory and sent to UCSF for testing. For NET staining, slides were stained with commercially available NET antibody (NET17-1; MAb Technologies; Stone Mountain, GA) at a 1:2000 dilution. Antigen was detected using an avidin:biotinylated enzyme complex (ABC detection; Commercial Kit). Positive controls included pheochromocytoma and human embryonic kidney (HEK) cells transfected with NET. Negative controls included pheochromocytoma with no primary antibody added as well as tonsil.

For VMAT1 staining, slides were heated at $60^{\circ} \mathrm{C}$ for 30 minutes prior to staining. They were then deparaffinized and rehydrated, then placed in a pressure cooker in $10 \mathrm{mM}$ citrate buffer. VMAT1 rabbit polyclonal antibody (Phoenix Pharmaceuticals \#H-V001; 1:500 Dilution) was incubated with slides overnight at $4^{\circ} \mathrm{C}$, and then a HRP-conjugated secondary antibody was incubated for 30 minutes at room temperature. Lastly, the slides were incubated with diaminobenzidine using a high-sensitivity substrate chromogen system (Dako; Carpinteria, CA). Positive controls included pheochromocytoma and HEK cells transfected to express VMAT1. Negative controls included tonsil, HEK cells transfected with empty vector, HEK cells transfected to express VMAT1 with no primary antibody added, and pheochromocytoma with no primary antibody added.

For VMAT2 staining, slides were prepared and stained as for VMAT1 except a VMAT2 mouse IgG1 monoclonal antibody (Origene\# TA500506; Clone 9E11; 1:400 dilution) was used. Positive controls included pheochromocytoma and HEK cells transfected to express VMAT2. Negative controls included tonsil, HEK with empty vector, HEK cells transfected to express VMAT2 with no primary antibody added, and pheochromocytoma with no primary antibody added.

Each slide was reviewed by two of three investigators (a pediatric pathologist, GK, for all slides and either WT or LM as second reviewer), with consensus results used for analysis. Investigators were blinded to patient clinical and biological features at the time of slide review. Stains were assigned a semi-quantitative intensity value of 0 to $3+$ for tumor cell staining. In the event of heterogeneous staining, the higher intensity category was coded as long as at least $5 \%$ of cells stained in this category. The percent of tumor cells expressing the protein of interest was also recorded. A composite protein expression score was calculated as the simple product of the intensity score times the percent of positive tumor cells.

\section{Statistical Analyses}

We used a series of Wilcoxon rank-sum tests to compare VMAT1 or VMAT2 composite protein scores between patient groups defined by clinical and biological features of interest including MIBG avidity (avid vs. non-avid), age at diagnosis (dichotomized at 18 months), primary site (adrenal vs. other), $M Y C N$ status (amplified vs. not amplified), tumor ploidy (hypodiploid or diploid vs. hyperdiploid), International Neuroblastoma Pathology 
Classification (INPC) histology classification (favorable vs. unfavorable) [18], mitotic karyorrhectic index (MKI; low/intermediate or $0-4 \%$ vs. high or $>4 \%$ ), tumor grade (differentiating vs. poorly differentiated or undifferentiated), catecholamine status at diagnosis (elevated vs. not elevated), 1p status [loss of heterozygosity (LOH) vs. no LOH], and 11q status (aberration vs. no aberration).

As we had a particular interest in the association of VMAT expression with MIBG avidity, we also used Wilcoxon rank-sum tests to compare percent tumor cells positive for VMAT1 or VMAT2 between MIBG avid and MIBG non-avid tumors. We also used Fisher exact tests to determine whether VMAT protein expression intensity categories were associated with MIBG avidity.

The degree of statistical dependence between NET and VMAT1 and VMAT2 levels was assessed by calculating Spearman rank correlation coefficients. Scatter plots with best-fit lines were produced to accompany these correlative tests.

\section{Results}

\section{VMAT1 and VMAT2 Expression in Neuroblastoma Cell Lines}

We first assessed the range of VMAT1 and VMAT2 protein expression in neuroblastoma cell lines (Fig 1). All cell lines expressed VMAT1, a subset expressed VMAT2, and the majority expressed NET. There was not a clear pattern of expression based on MYCN amplification status, MYC protein expression, or NET expression.

\section{VMAT1 and VMAT2 Expression in Neuroblastoma Tumors}

Of the 82 patients evaluated, 6 patients had samples with inadequate tumor material for assessment of VMAT1 or VMAT2 expression, due to crush artifact, necrotic tumor, or insufficient tumor cells. Characteristics of the 76 patients with evaluable samples are shown in Table 1.

VMAT1 and VMAT2 were expressed in $62 \%$ and $75 \%$ of neuroblastoma tumors, respectively (see Supplemental Fig 1 for representative IHC images). VMAT2 staining intensity was $3+$ in $55 \%$ of tumors compared to $32 \%$ for VMAT1. The distribution of VMAT1 and VMAT2 composite protein expression scores is shown in Figure 2a. Both proteins showed a wide range of expression between individual tumors. The median VMAT2 composite expression score was higher than the median VMAT1 composite expression score (153 vs. 47). VMAT1 and VMAT2 expression scores showed moderate correlation (Supplemental Fig 2; correlation coefficient 0.66; $\mathrm{p}<0.001$ ), though there were tumors with intense VMAT2 expression that did not express VMAT1.

\section{VMAT1 and VMAT2 Expression Correlate with Clinical and Biological Features in Patients with High-Risk Neuroblastoma}

Correlation of VMAT1 and VMAT2 composite protein expression scores with clinical and biological features is shown in Table 1. VMAT1 and VMAT2 protein expression scores were significantly lower in MYCN amplified tumors and in tumors with high MKI. In addition, VMAT2 protein expression scores were significantly lower in hypodiploid / 
diploid tumors and tumors without $11 \mathrm{q}$ aberration. There was a trend to suggest lower VMAT2 expression in tumors from patients $<18$ months of age at diagnosis and in nonadrenal primary tumors.

\section{VMAT2 Expression Correlates with MIBG Avidity}

Given their role as monoamine transporters, we were particularly interested to evaluate whether VMAT1 and/or VMAT2 protein expression correlate with MIBG avidity based upon diagnostic MIBG scans. VMAT1 expression intensity category, percent of cells positive for VMAT1, and VMAT1 composite expression score were not associated with MIBG avidity (Table 2 and Fig 2b). For VMAT2, expression intensity category was not statistically associated with MIBG avidity. However, MIBG avid tumors had significantly higher VMAT2 composite expression scores compared to MIBG non-avid tumors, though there was substantial overlap between groups (median 216 vs. 45; $p=0.04$; Table 2 and Fig 2c). We also observed a trend to suggest that MIBG avid tumors had a higher percent of cells positive for VMAT2 protein expression compared to MIBG non-avid tumors (median $74 \%$ vs. $18 \% ; \mathrm{p}=0.05)$.

\section{VMAT1 and VMAT2 Expression Correlate with NET Expression}

As NET protein expression plays a key role in MIBG avidity, we next evaluated whether VMAT1 and VMAT2 protein expression correlate with NET protein expression. We observed a weak correlation between VMAT1 and NET protein expression, though all of the tumors with intense VMAT1 expression had at least moderate NET expression (Fig 3a; correlation coefficient $0.46 ; p<0.001$ ). There was a greater degree of correlation between VMAT2 and NET protein expression, particularly at the upper end of expression (Fig 3b; correlation coefficient $0.67 ; \mathrm{p}<0.001$ ).

Our previous work demonstrated that $M Y C N$ amplified tumors showed lower NET protein expression compared to $M Y C N$ non-amplified tumors.[10] The current project allowed us an opportunity to validate this finding in an independent data set (with only one MIBG nonavid patient in common with our prior analysis). We again observed that $M Y C N$ amplified tumors had lower NET composite protein expression scores compared to MYCN nonamplified tumors (median 80 vs. 195; $\mathrm{p}=0.007$ ).

\section{Discussion}

To our knowledge, this is the first study to examine the correlation between the expression of the two VMAT isoforms and MIBG avidity in human neuroblastoma tumors. We report a positive correlation between VMAT2 expression and MIBG tumor avidity. We found no association between VMAT1 protein expression and MIBG tumor avidity. Our investigations also revealed several associations between other features of neuroblastoma and VMAT protein expression. VMAT1 and VMAT2 proteins had significantly lower levels of expression in neuroblastoma tumors with $M Y C N$ amplification and in tumors with high MKI.

NET has traditionally been thought to be the primary transporter responsible for MIBG uptake. Our group previously found a positive correlation between NET expression and 
MIBG avidity in neuroblastoma tumors, though there was substantial overlap between NET protein expression levels in MIBG avid and non-avid tumors [10]. This finding suggested the possibility that NET may not be the only protein mediating MIBG avidity. Previous studies have already implicated VMAT1 and VMAT2 in MIBG transport in other neuroendocrine tumors $[15,14]$. Until now, this phenomenon has remained unstudied in clinical cases of neuroblastoma. The positive statistical association we have observed between VMAT2 expression and MIBG avidity is not only consistent with our current understanding of the function of VMAT in neuroendocrine tumors, but also suggests that VMAT2 may also mediate MIBG avidity in neuroblastoma tumors.

Our investigations have also revealed strong correlations between VMAT expression and other features of neuroblastoma. While we have previously shown that MYCN amplified tumors have lower NET protein expression [10], until now, no reports have evaluated the relationship between VMAT protein expression and tumor biology. Here, we found a significant association between MYCN amplification and low levels of VMAT1 and 2 expression. Taken together with our previous findings, these results suggest a general pattern of lower membrane transporter expression in MYCN amplified tumors, perhaps as a marker of overall reduced neural differentiation in these tumors. Given this pattern, one might hypothesize that $M Y C N$ amplified tumors are more likely to be MIBG non-avid, though, to our knowledge, there are no data to support this hypothesis.

We also found lower VMAT protein expression in tumors with high MKI. VMAT2 protein expression was also significantly higher in hyperdiploid tumors and in tumors with an 11q aberration. We observed a trend toward higher VMAT2 protein expression in adrenal primary tumors. VMAT1 and 2 are known to be highly expressed in normal adrenal medullary tissue [19-21], though this expression in normal tissue should not have impacted our results in adrenal neuroblastoma as only expression on tumor cells was scored in our study. It is unlikely that this pattern was confounded by the association between MYCN amplification and VMAT2 expression as adrenal primary tumors have the highest rates of MYCN amplification [22], a pattern which should lead to overall lower levels of VMAT2 protein in adrenal tumors. In contrast, it is possible that the other observed associations between VMAT expression and MKI, ploidy, and 11q status are due to the known effect of $M Y C N$ status on these features [23, 24].

We acknowledge certain limitations in our work. We provide statistical correlations between VMAT expression and clinical / biological features of high-risk neuroblastoma. Future studies will be required to examine possible mechanisms behind these associations to determine if they are causally linked. Although we did find statistically significant correlations between VMAT expression, MIBG avidity and aspects of tumor biology in neuroblastoma, we also note a wide range of VMAT1 and 2 expression resulting in substantial overlap between groups. It is not clear if this overlap is a function of the immunohistochemistry assays or represents the underlying heterogeneity of these tumors. Furthermore, since VMAT1 and VMAT2 expression both positively correlate with NET expression, we concede the possibility that our results may be due to the relationship between NET, neuroblastoma tumor biology and MIBG avidity. In addition, as our patient population was chosen specifically to target high-risk, stage 4 disease patients, as this is the 
targeted population for MIBG therapy, we are unable to determine whether these results will generalize to low, intermediate-risk or localized high-risk disease patients. We evaluated only one tumor per patient and cannot speak to whether the results in that tumor are representative of all of the patient's sites of disease involvement. Likewise, within a tumor itself, we were not able to determine the site from which the biopsy was obtained and whether that portion of the tumor showed avidity for MIBG. As these proteins may be expressed heterogeneously throughout at tumor, it is possible that we may have analyzed some tumor samples that were partially MIBG-non avid. Despite these limitations, our work is novel and we describe new correlations between VMAT expression and clinical and biological features in neuroblastoma. Our study benefited from a uniform patient population (all patients had high-risk, stage 4 disease), and a relatively large sample size given the rarity of neuroblastoma. The use of multiple reviewers to grade immunohistochemistry results as well as uniform central review of all MIBG scans both contribute to the quality of our data.

Our work should motivate future studies of several key areas. Future studies will need to investigate potential mechanistic link between VMAT2 expression and MIBG avidity, including whether VMAT2 mediates primary MIBG transport, MIBG retention in tumor cells, or a combination of both roles. Moreover, the interaction between MYCN status and expression of other markers of neural differentiation merits further investigation. Studies investigating the role of signaling pathways, transcriptional factors, and post-translational factors important in the regulation of the VMAT proteins will be key to understanding why expression of these transporters is linked to other aspects of neuroblastoma tumor biology. From a clinical perspective, it will be of interest to evaluate whether VMAT2 expression correlates with response to MIBG therapy. Such studies will yield results important to expanding our current understanding of how MIBG therapy works, and improving this therapy in neuroblastoma patients.

\section{Supplementary Material}

Refer to Web version on PubMed Central for supplementary material.

\section{Acknowledgments}

Funding: Supported by the Campini Foundation (SGD and KKM), Dougherty Family Foundation (KKM), Alex's Lemonade Stand Foundation (WT, SGD, and KKM), U10CA09853, U10CA180899, and U24CA114766. The funding sources did not play a role in study design, conduct, data analysis, or interpretation.

\section{References}

1. Maris JM, Hogarty MD, Bagatell R, Cohn SL. Neuroblastoma. Lancet. 2007; 369(9579):2106-20. [PubMed: 17586306]

2. Taggart D, Dubois S, Matthay KK. Radiolabeled metaiodobenzylguanidine for imaging and therapy of neuroblastoma. Q J Nucl Med Mol Imaging. 2008; 52(4):403-18. [PubMed: 19088694]

3. DuBois SG, Matthay KK. Radiolabeled metaiodobenzylguanidine for the treatment of neuroblastoma. Nucl Med Biol. 2008; 35(Suppl 1):S35-48. [PubMed: 18707633]

4. Wilson JS, Gains JE, Moroz V, Wheatley K, Gaze MN. A systematic review of 131I-meta iodobenzylguanidine molecular radiotherapy for neuroblastoma. Eur J Cancer. 2014; 50(4):801-15. [PubMed: 24333097] 
5. Treuner J, Feine U, Niethammer D, Muller-Schaumburg W, Meinke J, Eibach E, et al. Scintigraphic imaging of neuroblastoma with [131-I]iodobenzylguanidine. Lancet. 1984; 1(8372):333-4. [PubMed: 6141395]

6. Smets LA, Janssen M, Rutgers M, Ritzen K, Buttenhuis C. Pharmacokinetics and intracellular distribution of the tumor-targeted radiopharmaceutical m-iodobenzylguanidine in SK-N-SH neuroblastoma and PC-12 pheochromocytoma cells. International journal of cancer Journal international du cancer. 1991; 48(4):609-15. [PubMed: 2045205]

7. Smets LA, Loesberg C, Janssen M, Metwally EA, Huiskamp R. Active uptake and extravesicular storage of m-iodobenzylguanidine in human neuroblastoma SK-N-SH cells. Cancer Res. 1989; 49(11):2941-4. [PubMed: 2720653]

8. Smets LA, Rutgers M. Model studies on metaiodobenzylguanidine (MIBG) uptake and storage: relevance for 131I-MIBG therapy of neuroblastoma. Journal of nuclear biology and medicine. 1991; 35(4):191-4.

9. Carlin S, Mairs RJ, McCluskey AG, Tweddle DA, Sprigg A, Estlin C, et al. Development of a realtime polymerase chain reaction assay for prediction of the uptake of meta[(131)I]iodobenzylguanidine by neuroblastoma tumors. Clin Cancer Res. 2003; 9(9):3338-44. [PubMed: 12960120]

10. DuBois SG, Geier E, Batra V, Yee SW, Neuhaus J, Segal M, et al. Evaluation of Norepinephrine Transporter Expression and Metaiodobenzylguanidine Avidity in Neuroblastoma: A Report from the Children's Oncology Group. Int J Mol Imaging. 2012; 2012:250834. [PubMed: 23050139]

11. Mairs RJ, Livingstone A, Gaze MN, Wheldon TE, Barrett A. Prediction of accumulation of 131Ilabelled meta-iodobenzylguanidine in neuroblastoma cell lines by means of reverse transcription and polymerase chain reaction. Br J Cancer. 1994; 70(1):97-101. [PubMed: 7517173]

12. Lawal HO, Krantz DE. SLC18: Vesicular neurotransmitter transporters for monoamines and acetylcholine. Molecular aspects of medicine. 2013; 34(2-3):360-72. [PubMed: 23506877]

13. Guilloteau D, Baulieu JL, Huguet F, Viel C, Chambon C, Valat C, et al. Meta-iodobenzylguanidine adrenal medulla localization: autoradiographic and pharmacologic studies. Eur J Nucl Med. 1984; 9(6):278-81. [PubMed: 6745297]

14. Kolby L, Bernhardt P, Levin-Jakobsen AM, Johanson V, Wangberg B, Ahlman H, et al. Uptake of meta-iodobenzylguanidine in neuroendocrine tumours is mediated by vesicular monoamine transporters. Br J Cancer. 2003; 89(7):1383-8. [PubMed: 14520475]

15. Fottner C, Helisch A, Anlauf M, Rossmann H, Musholt TJ, Kreft A, et al. 6-18F-fluoro-Ldihydroxyphenylalanine positron emission tomography is superior to 123I-metaiodobenzylguanidine scintigraphy in the detection of extraadrenal and hereditary pheochromocytomas and paragangliomas: correlation with vesicular monoamine transporter expression. J Clin Endocrinol Metab. 2010; 95(6):2800-10. [PubMed: 20371665]

16. Kreissman SG, Seeger RC, Matthay KK, London WB, Sposto R, Grupp SA, et al. Purged versus non-purged peripheral blood stem-cell transplantation for high-risk neuroblastoma (COG A3973): a randomised phase 3 trial. The Lancet Oncology. 2013; 14(10):999-1008. [PubMed: 23890779]

17. Yanik GA, Parisi MT, Shulkin BL, Naranjo A, Kreissman SG, London WB, et al. Semiquantitative mIBG scoring as a prognostic indicator in patients with stage 4 neuroblastoma: a report from the Children's oncology group. J Nucl Med. 2013; 54(4):541-8. [PubMed: 23440556]

18. Shimada H, Ambros IM, Dehner LP, Hata J, Joshi VV, Roald B, et al. The International Neuroblastoma Pathology Classification (the Shimada system). Cancer. 1999; 86(2):364-72. [PubMed: 10421273]

19. Anlauf M, Eissele R, Schafer MK, Eiden LE, Arnold R, Pauser U, et al. Expression of the two isoforms of the vesicular monoamine transporter (VMAT1 and VMAT2) in the endocrine pancreas and pancreatic endocrine tumors. The journal of histochemistry and cytochemistry : official journal of the Histochemistry Society. 2003; 51(8):1027-40. [PubMed: 12871984]

20. Erickson JD, Schafer MK, Bonner TI, Eiden LE, Weihe E. Distinct pharmacological properties and distribution in neurons and endocrine cells of two isoforms of the human vesicular monoamine transporter. Proceedings of the National Academy of Sciences of the United States of America. 1996; 93(10):5166-71. [PubMed: 8643547] 
21. Peter D, Liu Y, Sternini C, de Giorgio R, Brecha N, Edwards RH. Differential expression of two vesicular monoamine transporters. The Journal of neuroscience : the official journal of the Society for Neuroscience. 1995; 15(9):6179-88. [PubMed: 7666200]

22. Vo KT, Matthay KK, Neuhaus J, London WB, Hero B, Ambros PF, et al. Clinical, biologic, and prognostic differences on the basis of primary tumor site in neuroblastoma: a report from the international neuroblastoma risk group project. J Clin Oncol. 2014; 32(28):3169-76. [PubMed: 25154816]

23. Ambros PF, Ambros IM, Brodeur GM, Haber M, Khan J, Nakagawara A, et al. International consensus for neuroblastoma molecular diagnostics: report from the International Neuroblastoma Risk Group (INRG) Biology Committee. Br J Cancer. 2009; 100(9):1471-82. [PubMed: 19401703]

24. Goto S, Umehara S, Gerbing RB, Stram DO, Brodeur GM, Seeger RC, et al. Histopathology (International Neuroblastoma Pathology Classification) and MYCN status in patients with peripheral neuroblastic tumors: a report from the Children's Cancer Group. Cancer. 2001; 92(10): 2699-708. [PubMed: 11745206] 

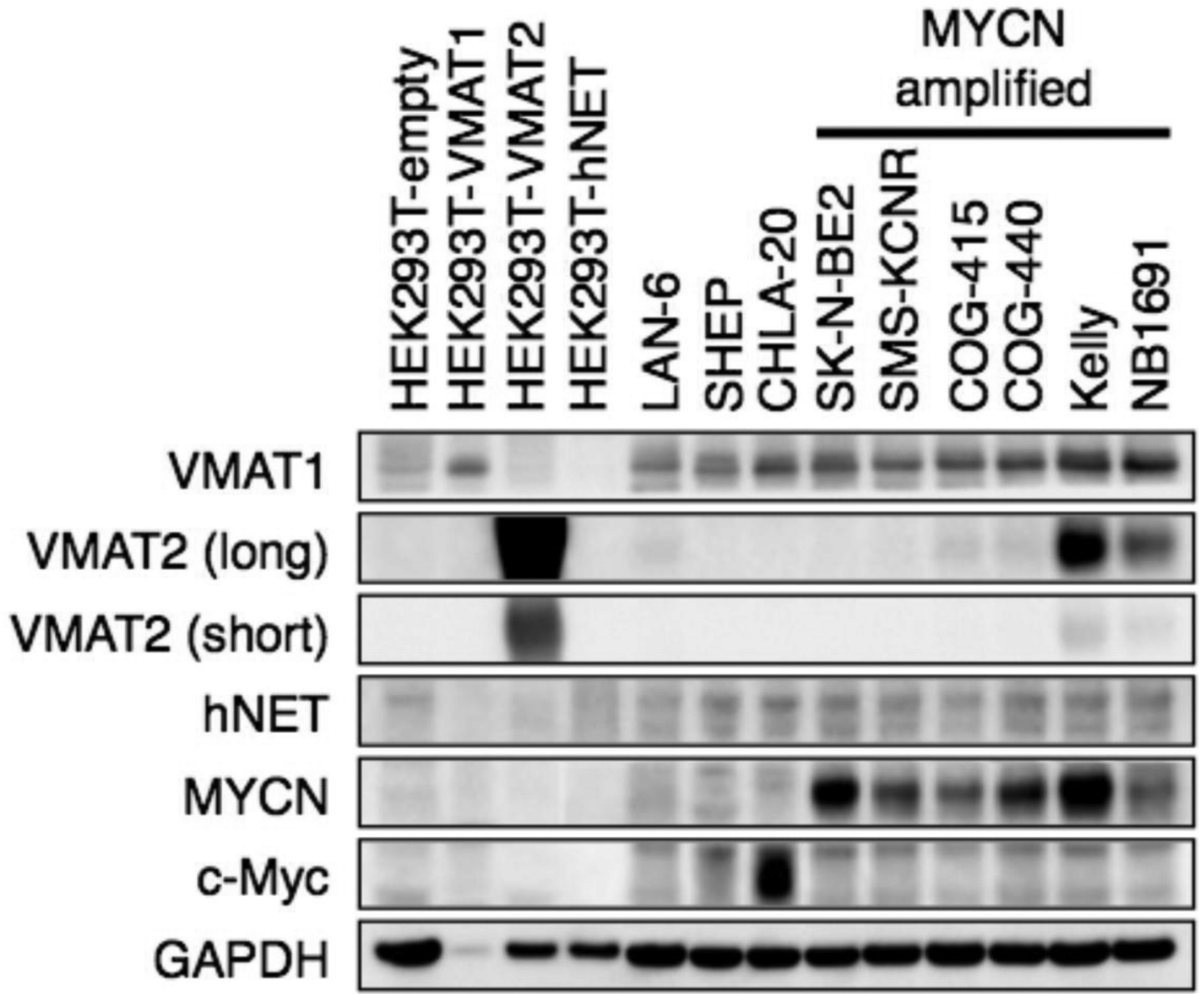

Fig 1.

VMAT1 and VMAT2 proteins are expressed in human neuroblastoma cell lines. Western blot demonstrating VMAT1, VMAT2, and NET protein expression in a panel of neuroblastoma and control cell lines. MYCN amplification status, MYCN protein expression, and MYC protein expression shown for reference. 
A

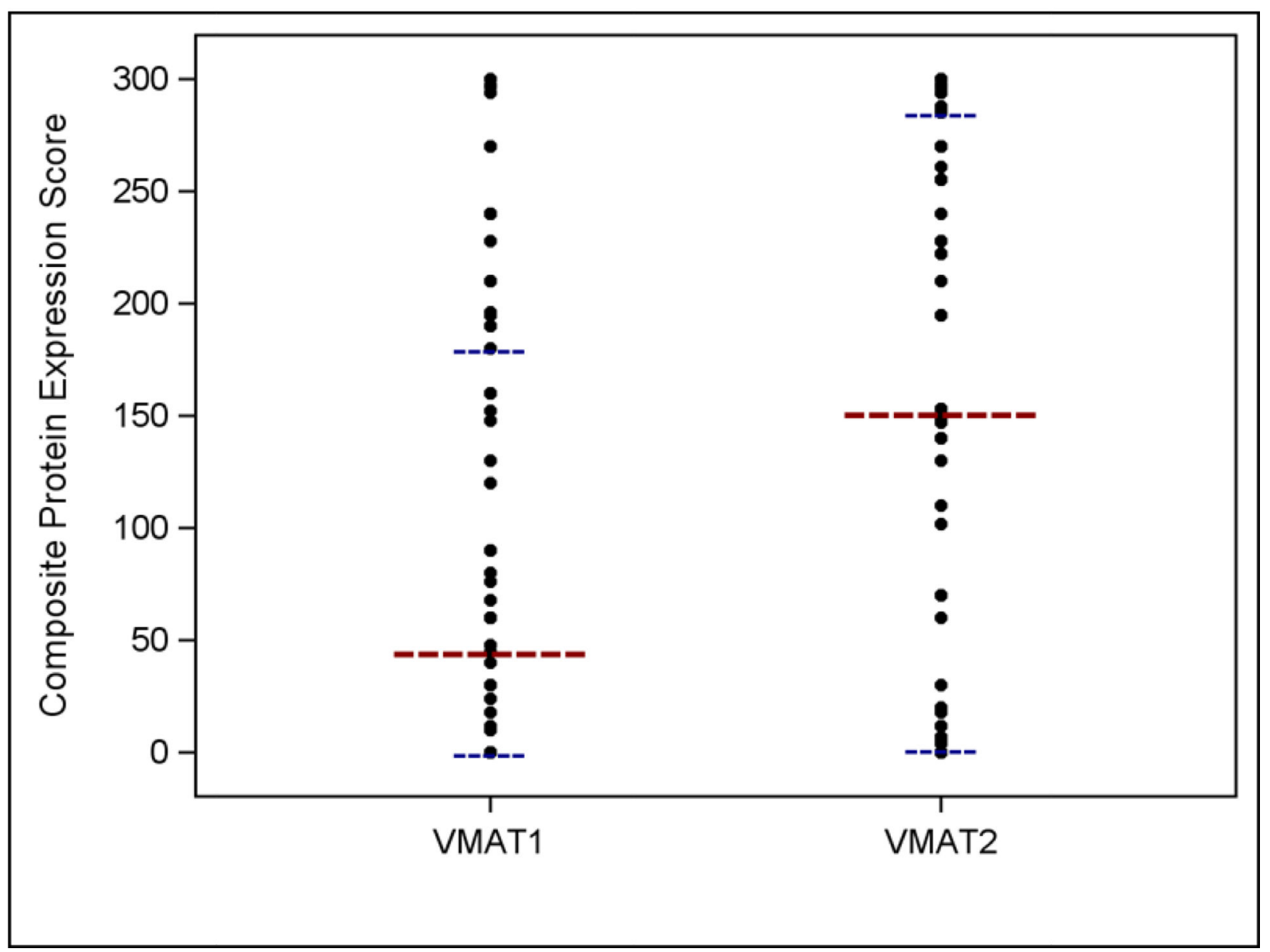

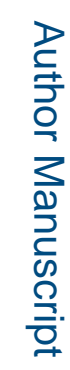


B

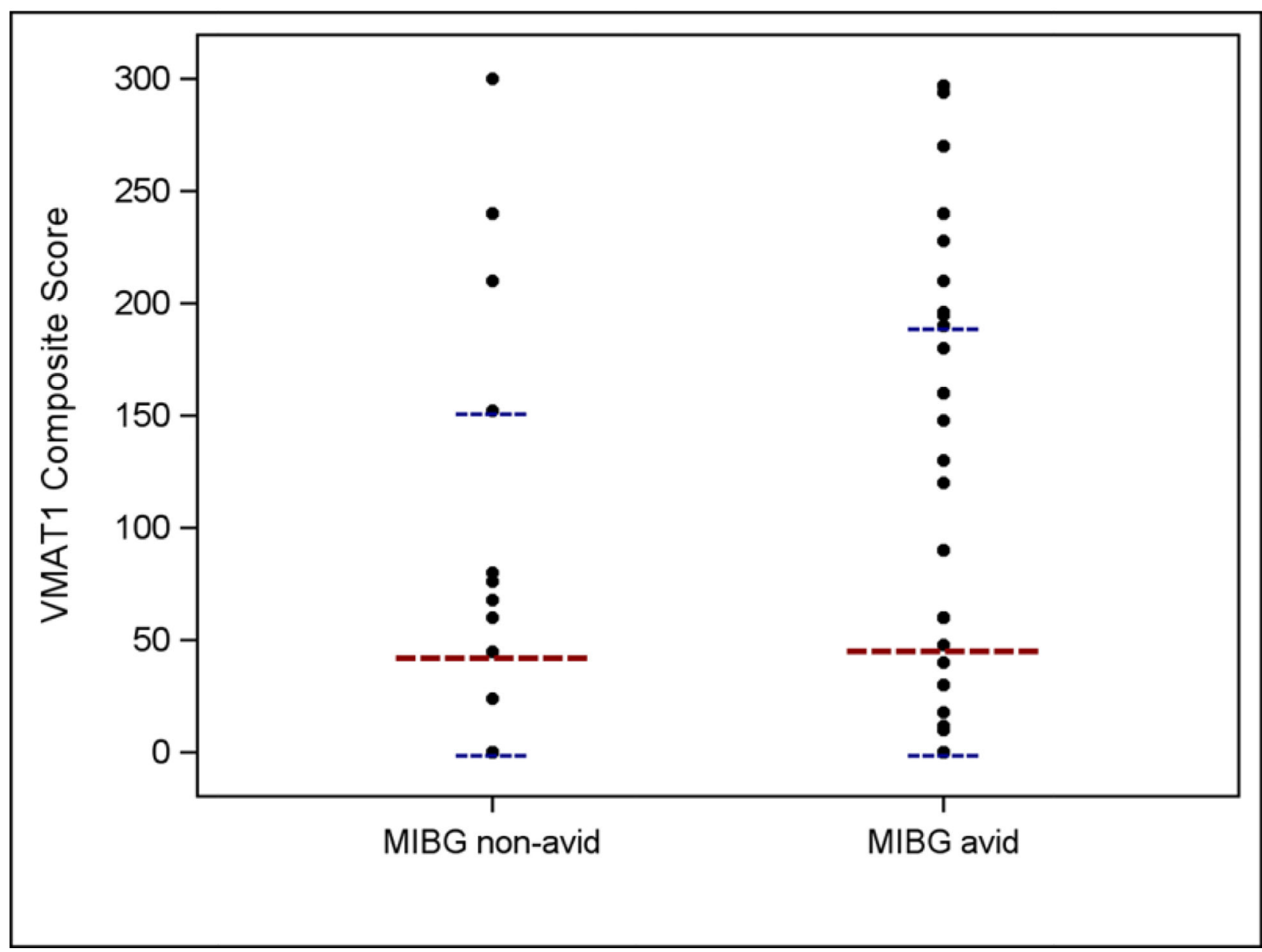

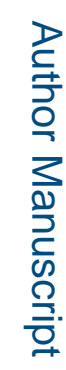


C

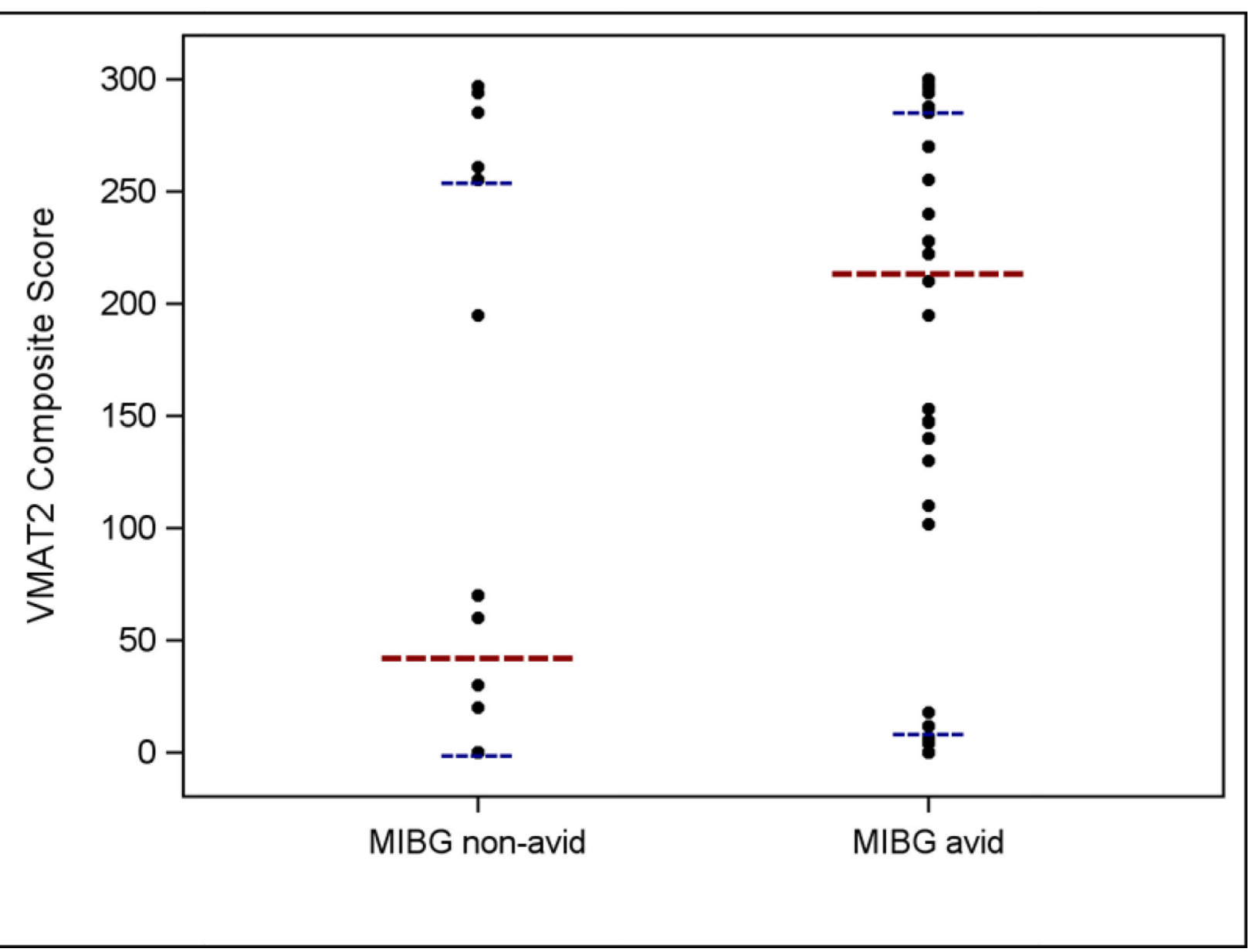

Fig 2.

a Distribution of VMAT1 and VMAT2 composite protein expression scores in human neuroblastoma tumor samples ( $\mathrm{n}=74$ for VMAT1 and $\mathrm{n}=76$ for VMAT2). b VMAT1 composite protein expression scores according to MIBG avidity in patients with neuroblastoma $(\mathrm{p}=0.76)$. $\mathbf{c}$ VMAT2 composite protein expression scores according to MIBG avidity in patients with neuroblastoma $(\mathrm{p}=0.04)$. For all panels, the longer horizontal dashed lines indicate group medians and the shorter horizontal dashed lines indicate $25^{\text {th }}$ and $75^{\text {th }}$ percentiles. 


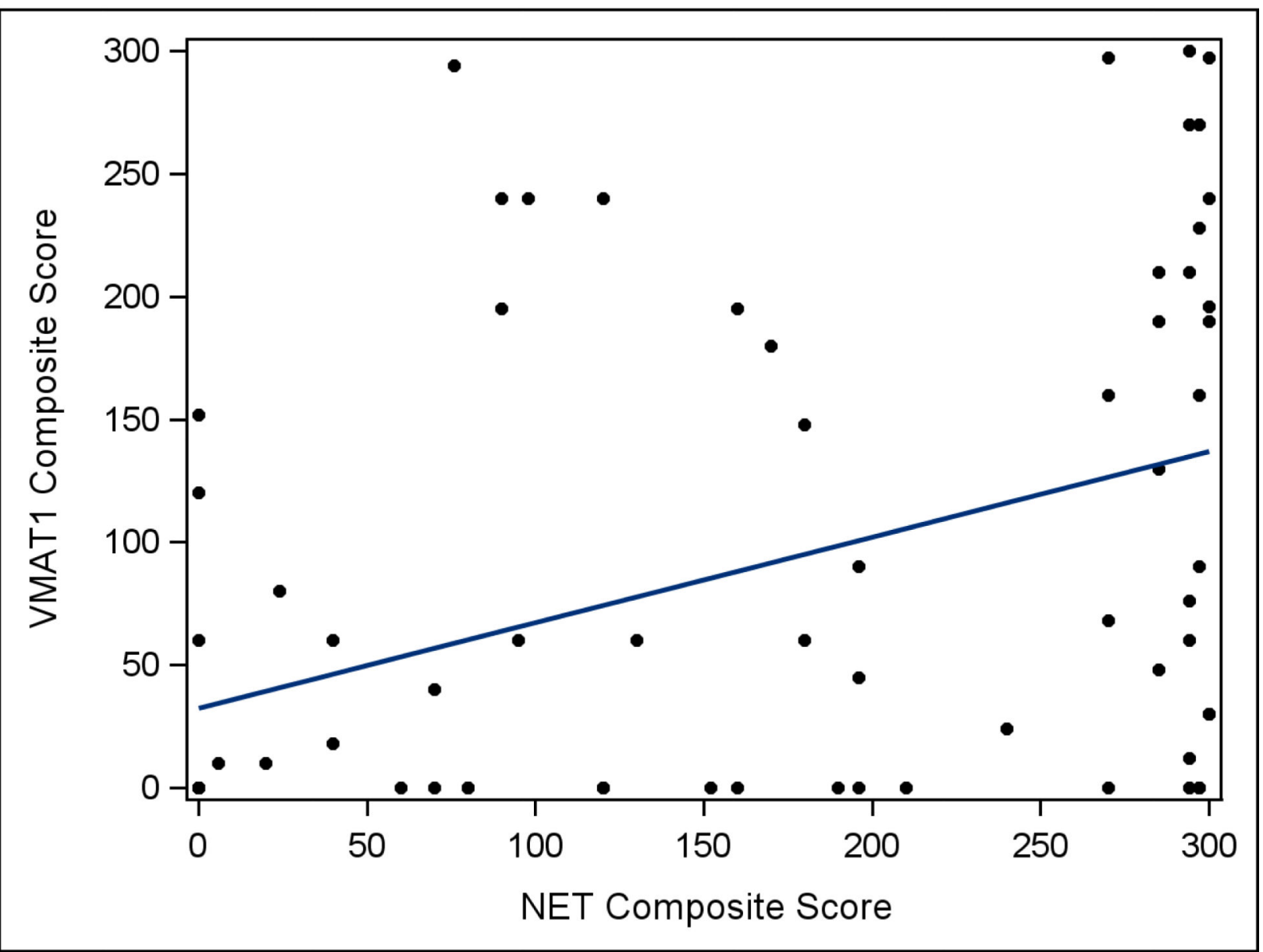


B

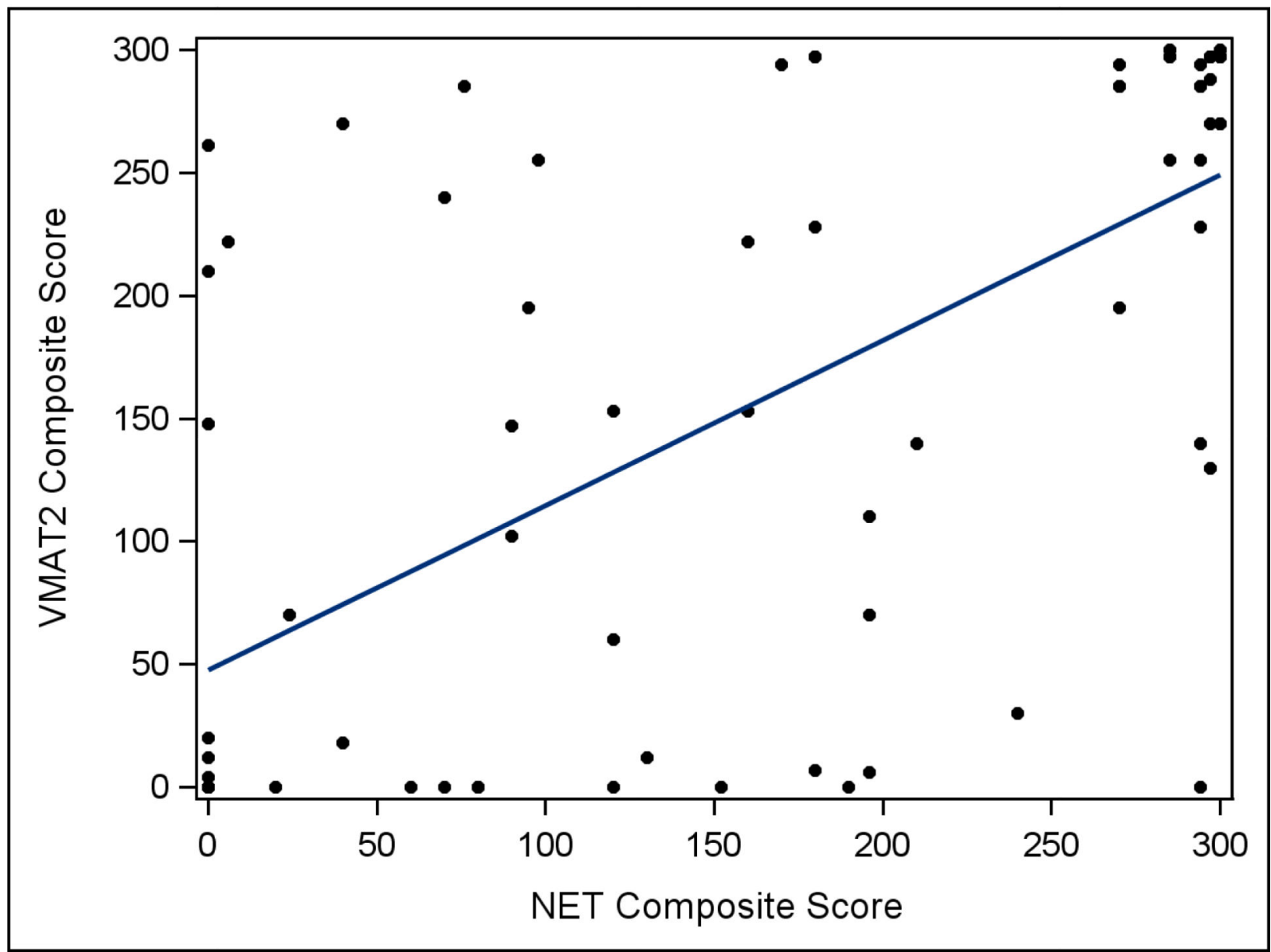

Fig 3.

a Correlation between VMAT1 composite protein expression score and NET composite protein expression score in human neuroblastoma tumors $(\mathrm{r}=0.46, \mathrm{p}<0.001)$. b Correlation between VMAT2 composite protein expression score and NET composite protein expression score in human neuroblastoma tumors $(\mathrm{r}=0.67, \mathrm{p}<0.001)$. 
Table 1

Clinical and biological features and corresponding VMAT1 and VMAT2 expression in patients with high-risk neuroblastoma.

\begin{tabular}{|c|c|c|c|c|c|}
\hline Characteristic & Overall N (\%) & $\begin{array}{c}\text { Median VMAT1 Composite } \\
\text { Score }^{a}\end{array}$ & p-value & $\begin{array}{c}\text { Median VMAT2 Composite } \\
\text { Score }^{a}\end{array}$ & p-value \\
\hline Age $\geq 18$ months & $66(86.8)$ & 60 & 0.15 & 203 & 0.08 \\
\hline Age $<18$ months & $10(13.2)$ & 0 & & 10 & \\
\hline MYCN Amplified & $28(41.8)$ & 0 & 0.003 & 5 & $<0.001$ \\
\hline$M Y C N$ Non-Amplified & $39(58.2)$ & 90 & & 270 & \\
\hline Adrenal Primary & $43(56.6)$ & 54 & 0.56 & 210 & 0.07 \\
\hline Non-Adrenal Primary & $33(43.4)$ & 38 & & 102 & \\
\hline Catecholamine Positive & $61(83.6)$ & 60 & 0.12 & 195 & 0.11 \\
\hline Catecholamine Negative & $12(16.4)$ & 0 & & 2 & \\
\hline Unfavorable Histology & $71(95.9)$ & 54 & 0.82 & 153 & 0.19 \\
\hline Favorable Histology & $3(4.1)$ & 83 & & 297 & \\
\hline Un/Poorly Differentiated & $59(89.4)$ & 35 & 0.48 & 153 & 0.74 \\
\hline Differentiating & $7(10.6)$ & 79 & & 195 & \\
\hline High MKI & $24(36.4)$ & 0 & 0.03 & 0 & $<0.001$ \\
\hline Low/Intermediate MKI & $42(63.6)$ & 60 & & 248 & \\
\hline Hypodiploid/Diploid & $39(57.4)$ & 10 & 0.07 & 30 & 0.009 \\
\hline Hyperdiploid & $29(42.6)$ & 74 & & 240 & \\
\hline 11q Aberration & $6(42.9)$ & 140 & 0.10 & 293 & 0.02 \\
\hline No 11q Aberration & $8(57.1)$ & 23 & & 70 & \\
\hline $1 \mathrm{p} \mathrm{LOH}$ & $5(33.3)$ & 0 & 0.35 & 0 & 0.14 \\
\hline No $1 p \mathrm{LOH}$ & $10(66.6)$ & 85 & & 263 & \\
\hline
\end{tabular}

${ }^{a}$ Composite score is product of intensity score and percent tumor cells positive. VMAT1 data for 74 patients. VMAT2 data for 76 patients. 
Table 2

Correlation of measures of VMAT1 and VMAT2 protein expression with MIBG avidity.

\begin{tabular}{|c|c|c|c|}
\hline & MIBG Avid $(\mathrm{n}=56)^{a}$ & MIBG Non-Avid $(\mathrm{n}=20)^{a}$ & p-value \\
\hline VMAT1 Expression Intensity Category & & & 0.91 \\
\hline $0+$ & $20(36.4 \%)$ & $8(42.1 \%)$ & \\
\hline $1+$ & $5(9.1 \%)$ & $2(10.5 \%)$ & \\
\hline $2+$ & $11(20.0 \%)$ & $4(21.1 \%)$ & \\
\hline $3+$ & $19(34.6 \%)$ & $5(26.3 \%)$ & \\
\hline Median Percent Cells Positive for VMAT1 (Range) & $20(0-99)$ & $24(0-100)$ & 0.80 \\
\hline Median VMAT1 Composite Expression Score (Range) & $48(0-297)$ & $45(0-300)$ & 0.76 \\
\hline VMAT2 Expression Intensity Category & & & 0.26 \\
\hline $0+$ & $11(19.6 \%)$ & $8(40 \%)$ & \\
\hline $1+$ & $3(5.4 \%)$ & $1(5 \%)$ & \\
\hline $2+$ & $8(14.3 \%)$ & $3(15 \%)$ & \\
\hline $3+$ & $34(60.7 \%)$ & $8(40 \%)$ & \\
\hline Median Percent Cells Positive for VMAT2 (Range) & $74(0-100)$ & $18(0-99)$ & 0.05 \\
\hline Median VMAT2 Composite Expression Score (Range) & $216(0-300)$ & $45(0-297)$ & 0.04 \\
\hline
\end{tabular}

${ }^{a}$ Data for VMAT1 expression not available for one patient with MIBG avid tumor and one patient with MIBG non-avid tumor. 